\title{
The Role of Chemotherapy in Triple Negative Breast Cancer
}

\author{
Almuradova DM* \\ Department of Tashkent Medical Academy, Uzbekistan \\ *Corresponding author: Almuradova DM, Department of Tashkent Medical Academy, Uzbekistan
}

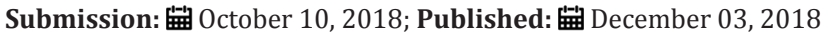

\begin{abstract}
Breast cancer (BC) is the most frequent tumor worldwide. Triple-negative BCs are characterized by the negative estrogen and progesterone receptors and negative HER2 and represent 12-18\% of all BCs.Breast cancer (breast cancer) is the most common oncological disease in women in the Uzbekistan. Data from epidemiological studies show that TNBC is more common in young women (up to 50 years) before menopause. In addition, the likelihood of TNBC is higher in women with early menarche, the first pregnancy at an earlier age, a short period of breastfeeding, and an increased body mass index. It should be not that the prognosis for TNBC does not depend on the degree of differentiation of the tumor, the presence of lymph node metastases, the size of the tumor or the treatment performed. The aggressiveness of TNBC was also confirm by the maximum risk of recurrence during the first three years after surgical treatment with a maximum mortality within the first 5 years. TNBC is a subtype of tumors with a special character of metastasis. Based on the above, we have undertaken the present work, having set ourselves the following goal: to improve immediate and long-term results of treatment of patients with locally advanced thyroid cancer by using the most effective diagnostic methods and comprehensive treatment.
\end{abstract}

Keywords: Triple negative BC; Neoadjuvant; Adjuvant; Metastatic chemotherapy; Paclitaxel; docetaxel; Gemcitabine

\section{Introduction}

Triple-negative breast cancers have a relapse pattern that is very different from hormone-positive breast cancers: the risk of relapse is much higher for the first 3-5 years but drops sharply and substantially below after that hormone-positive breast cancers. In this review, data on the use of gemcitabine in metastatic triplenegative BCs are analyzed, conclu ding they are effective in any clinical setting (neoadjuvant, adjuvant, and metastatic). The available data show the clinical potential of based combinations in terms of longduration response, increased survival, and better quality of life of patients with triple-negative metastatic BC. The ongoing trials will give further information on the better management of this type of tumor. In 2016, 1,380,000 new cases and 458,000 deaths for BC were reported worldwide, of which there were 332,000 new cases and 79,000 deaths in Asia [1-4]. BC in the territory of the Republic among urban and rural population by determining the average annual intensive and standardized incidence rates for the years 2008-2010. The highest standardized incidence rates registered in Tashkent city (22.5\%x>00), Navami (12.4\%), Bukhara (11.1\%) and Tashkent regions (11.0\%) and lowest in Skandera (6.3\%) and Kasandra (7.5\%). On the territory of the Republic those in urban areas more often suffer from cancer of the breast $(13,2 \% x>00)$ than rural $(8,5 \%)$.

The highest incidence was within the age intervals 50-59$18.0 \%, 60-69-31.2 \%, 70$ years and older $-19.3 \%$. In the structure of mortality of the female population of Urform malignant neoplasms the greatest proportion is malignant breast tumors (20.4\%) [3-6]. According to Cancer Registry of the Republican Oncology scientific center (RONTS), in 2012 in the general structure of cancer of malignant neoplasms of women for patients with breast cancer (24.6\%) occupy the 1 st ranked place, ahead of patients with cervical cancer (12.4\%), (5.9\%), ovaries (5.0\%) and the uterus body (4.4\%) $[7,8]$. According to the Ministry of Health of Uzbekistan, the most common cancer in the country is breast cancer.

This type of cancer is diagnosed in 9.1 cases per 100 thousand of the population. According to experts, the hereditary factor is important in the development of breast cancer in women, it is on the maternal line. It is transmitted in $45-75 \%$ of cases, if there are mutations of BRSA 1-2 genes. To prevent breast cancer, women under the age of 50 years should undergo ultrasound of the breast. Women aged 50 years and older should undergo mammogram of the breast. Also, all the representatives of the fair sex need to conduct self-examination of the breast every month. At any suspicion of the presence of compaction in the mammary glands, consultation of a mammologist is necessary, noted in the Ministry of Health\%). Despite the improvement of the methods of diagnosing this pathology, more than $50 \%$ of primary patients annually turn to the oncologist in the III and IV stages of the disease. Purpose of the study is to evaluate the effectiveness of various chemotherapy regimens for neoadjuvant, adjuvant and palliative polychemotherapy (metastatic) in patients triple negative breast cancer with improved long-term treatment outcomes. 


\section{Materials and Methods of Research}

The object of our study will be patients with breast cancer with a triple negative phenotype. In total, retrospective and prospective groups of patients treated in 2012-2017 will be studied. in Tashkent City oncology in the department of oncology and chemotherapy ( $n=99)$, patients with triple negative breast cancer. Thus, the patients included in the study were characterized by a set of unfavorable signs: in $93 \%$ of cases the tumors were characterized by high Ki-67, in 53\%-87.7\% of cases-by II-IV grade of malignancy. Criteria for selecting patients: Progression of breast cancer was detected in the period from 12 to 60 months after the operative removal of the primary tumor (metachronous metastases); ECOG $0-1$. The age of patients older than 18 years. The presence of the result of immunohistochemical analysis of the primary tumor, and in the experimental group a comparative analysis of the primary

\section{Result}

\section{The triple-negative BCs (TNBCs)}

Table 1: General characteristics of triple-negative BC. tumor and distant metastases. Functional status according to ECOG (FS) was from 0 to 2 . All patients had measurable normal kidney and liver function, satisfactory parameters of general and biochemical blood tests (leukocytes $>4.5 \times 10^{9} / \mathrm{L}$, neutrophil $>2.0 \times 10^{9} / \mathrm{L}$, hemoglobin $>9 \mathrm{~g} / \mathrm{dL}$, blood transfusions were not tolerated for the last two weeks, platelets $>100 \times 10^{9} / \mathrm{L}$, creatinine $<130 \mu \mathrm{mol} / \mathrm{L}$, total bilirubin $<1.5$ of the upper limit of normal (CGI), ALT and ACT $<1.5$ VGN. After randomization, patients in a 2:1 ratio were included in two treatment groups:

A. Chemotherapy with doxorubicin,

B. Chemotherapy with platinum drugs.

As chemotherapy, the following options were used: taxanes (docetaxel or paclitaxel), platinum, anthracyclines, gemcitabine, doxorubicin, cyclophosphane, capecitabine.

\begin{tabular}{|c|}
\hline Weak Association between Tumor Size and Lymph Node Involvement \\
\hline Quick growth and tissular density similar to normal tissue \\
\hline High expression of BRCA1 mutation \\
\hline High risk of early relapse \\
\hline Peak recurrence between the first and third years after diagnosis \\
\hline Metastases are rarely preceded by local recurrence \\
\hline Higher incidence in younger women, Afro American or Hispanic, and in low socioeconomic conditions \\
\hline Strongerassociationwithobesity \\
\hline Higher incidence of brain metastases \\
\hline Most deaths occur in the first 5 years \\
\hline Rapid progression from the onset of metastasis to death \\
\hline
\end{tabular}

The most important biological markers, not only for classification of $\mathrm{BC}$ but also for, the therapeutic strategy are the hormonal receptors (estrogen [ER] and progesterone [PgR] receptor) and the HER2 receptor status [5]. Tumors that are ER-, PgR-, and HER2-negative are known as TNBC and account for about $12-20 \%$ of BCs [4]. These tumors develop earlier in life, especially in premenopausal women, and have a poorer prognosis than the other types of $\mathrm{BC}$ due to the higher aggressiveness. These factors may be a major reason for the high-risk relapse, and shorter progression-free survival (PFS) and overall survival (OS) reported for this disease [3-6]. The main general characteristics of TNBC are summarized in Table 1.

Table 2:

\begin{tabular}{|c|c|c|}
\hline NCCN & ESMO & AIOM \\
\hline Monotherapy & Monotherapy (without Extensive Visceral Involvement/Symptomatic) & Monotherapy \\
\hline Paclitaxel & Weekly paclitaxel & Paclitaxel \\
\hline Docetaxel & Weekly docetaxel or q3w & Docetaxel \\
\hline Nab-paclitaxel & Nab-paclitaxel & Nab-paclitaxel \\
\hline Combinations & Combinations (with Extensive Visceral Involvement/Symptomatic) & Combinations \\
\hline Docetaxel+ capecitabine & Anthracycline+taxane (paclitaxel or docetaxel) & $\begin{array}{l}\text { Anthracycline }+ \text { taxane } \\
\text { (paclitaxel or docetaxel) }\end{array}$ \\
\hline Gemcitabine+ paclitaxel & Docetaxel + capecitabine & $\begin{array}{l}\text { Docetaxel + capecitabine } \\
\text { clitaxel + gemcitabine }\end{array}$ \\
\hline Paclitaxel+bevacizumab & Paclitaxel + carboplatin & $\begin{array}{l}\text { Docetaxel + gemcitabine } \\
\text { Paclitaxel + bevacizumab }\end{array}$ \\
\hline
\end{tabular}

Abbreviations: AIOM: Association Italian di Oncologic Medical; ESMO: European Society for Medical Oncology; NCCN: National Comprehensive Cancer Network; Q3W: Every 3 Weeks; BC: Breast Cancer 
TNBC is not a unique clinical entity. It comprises several types of cancers now characterized by molecular profiles, which represent different diseases with probably different treatment options and different response to chemotherapy, biological agents, and/or other therapeutic regimens. The gene expression assay classifies BCs into at least five groups, including luminal A, luminal B, HER2enriched, basal-like (BL), and normal breast-like. More recent gene expression array analysis has identified six different groups of TNBC, including two BLs (BL1 and BL2), an immune-modulatory (IM), a mesenchymal (M), a mesenchymal stem-like (MSL), and a luminal androgen receptor (LAR) subtype. BC subtypes array, 25\% of relapses were basal, 32\% HER2, 10\% luminal A, 28\% luminal $\mathrm{B}$, and $5 \%$ normal breast-like. Importantly, the intrinsic subtype at relapse was significantly associated with post relapse survival $(\mathrm{P}=0.012)$. At the 2016 on 1,100 women diagnosed in 2016 with TNBC in the Uzbekistan (Table 2). This population reflects the current clinical practice in the Uzbekistan at the time: $34 \%$ were at stage I, $42 \%$ at stage II, $15 \%$ at stage III, and $6 \%$ at stage IV, with a $24-$ month os of $97 \%, 93 \%, 71 \%$, and $27 \%$, respectively. The median OS in metastatic disease was 13 months [7-10].

\section{The treatment of TNBCs}

A proportion of TNBC is highly sensitive to chemotherapy but with a short PFS and a lower OS [11-15]. Current therapeutic strategies include chemotherapeutic drugs (anthracyclines, taxanes, platinum derivatives, and gemcitabine) and biological drug [16]. The efficacy of anthracyclines and gemcitabine in metastatic $\mathrm{BC}$ is higher in ER-negative tumors; for this reason, both classes are indicated as first-line treatment of TNBC, even if with a shortlasting benefit [14]. Another group of drugs with proven activity in TNBC are the platinum derivatives cisplatin and carboplatin [1316].

\section{Guidelines for the treatment of TNBCs}

There are no specific guidelines for the management of TNBC: The National Comprehensive Cancer Network (NCCN), European Society for Medical Oncology (ESMO), and Association Italian di Oncologic Medical (AIOM) Guidelines recommend that TNBC be treated with chemotherapy (monotherapy or combination therapy) but do not specify the most appropriate drugs. Taxanes and their combinations recommended by NCCN, ESMO, and AIOM guidelines for triple-negative BC.

The ESMO Guidelines states that cytotoxic chemotherapy is the standard of care for the treatment of TNBC and that the choice of the regimen should be made after consideration of disease-related factors (disease-free survival [DFS], previous therapies and response, tumor burden, and need for rapid disease/symptom control) and patient-related factors (patient preferences, biological age, menopausal status, comorbidities and performance status, and socioeconomic and psychological factors). Combination chemotherapy is more often required because of frequent visceral involvement, aggressive course, and risk of rapid patient deterioration. Finally, there is no a standard approach for chemotherapy after first line $[16,17]$.

\section{The role of taxanes in $\mathrm{TNBC}$}

The role of taxanes in TNBC is well established after the many studies evaluating the efficacy of taxane-based regimens in neoadjuvant, adjuvant, and metastatic disease settings. Neoadjuvant therapy has been used for a long time for reducing the size and the extension of locally advanced tumors, but now it is extensively used also in early $\mathrm{BC}$ not suitable for primary conservative surgery, with an added predictive value for the long-term outcome of the disease. The best efficacy target for neoadjuvant therapy is expressed as pathological complete response ( $\mathrm{PCR}$ ). The predictive value of pCR as a surrogate for long-term clinical benefit has been recently confirmed by the retrospective pooled analysis of Cortazar et al. [18]. This meta-analysis was based on the pCR, overall response rate (ORR), and event-free survival (EFS) data of 12 international clinical trials on a total 11,550 patients.

The analysis compared the three main definitions of pCR in order to establish their association with long-term efficacy: ypT0 ypN0 (no invasive and in situ tumor in the breast and auxiliary lymph nodes); ypT0/is ypN0 (no invasive tumor in the breast and auxiliary lymph nodes, independent of the presence of in situ ductal carcinoma); and ypT0/is ypN0/is (no invasive tumor in the breast, independent of the presence of in situ ductal carcinoma or lymph nodes involvement). The better combination between pCR and long-term effect was observed in patients with an aggressive tumor (TNBC; high-grade; ER/PgR-positive, HER2negative; HER2-positive; and ER- and PgR-negative) [19]. Several studies on neoadjuvant therapy confirmed the sensitivity of TNBC to cytotoxic drugs, as well as the importance of platinium-based chemotherapy. Rouzier et al. [20] evaluated the molecular-based chemosensitivity patients treated with carboplatin and taximen adjuvant therapy, and a pCR was observed in $45 \%$ of BL tumors and in $6 \%$ of luminal tumors (A and B) [21]. The study evaluated in 53 women randomized to neoadjuvant doxorubicin and docetaxel for four cycles or doxorubicin and cyclophosphamide (AC) for four cycles followed by docetaxel for four cycles. The overall was $10.6 \%$ ( $7 \%$ with the two-drug regimen and $14.3 \%$ with the triplet one). The efficacy of taxane-based neoadjuvant therapy was further confirmed by more recent trials. Wu et al Evaluated the efficacy of neoadjuvant therapy with docetaxel plus eirenicon, and the OS in 64 patients with TNBC and in 65 patients with non-TNBC. A pCR was observed in $25.9 \%$ of TNBCs, significantly higher than in the other subtypes $(\mathrm{P}=0.019)$ [22].

The New England Journal of Medicine published a report by Bear et al of another trial of neoadjuvant chemotherapy with or without carboplatin. This Phase III randomized trial assigned 46 patients with HER2-negative BC to receive docetaxel $\left(100 \mathrm{mg} / \mathrm{m}^{2}\right.$ every 21 days) or docetaxel $\left(75 \mathrm{mg} / \mathrm{m}^{2}\right.$ day 1$)$ plus capecitabine $\left(825 \mathrm{mg} / \mathrm{m}^{2}\right.$ twice a day 1 to 14$)$ or docetaxel $\left(75 \mathrm{mg} / \mathrm{m}^{2}\right.$ day 1$)$ plus gemcitabine $\left(1,000 \mathrm{mg} / \mathrm{m}^{2}\right.$ days 1 and 8$)$ for four cycles. All regimens were followed by AC for a further four cycles. All patients were also randomized to receive carboplatin $(A U C=5)$ or not for the first six cycles of chemotherapy. Results showed first of all that the addition of carboplatin and gemcitabine did not improve the rate of docetaxel alone and showed increased toxicity and that the toxicity 
of carboplatin was manageable and as expected from previous trials and, significantly increased the overall PCR rate (34.5\% vs $28.2 \%$ ) $(\mathrm{P}=0.02)$. The multiple logistic regression model showed that TNBC subtype, high grade, and smaller tumor size were associated with higher rates in the breast, but when considering the breast and nodes, the addition of carboplatin was significantly related to a better result in hormone receptor-positive tumors only.

In the randomized 45 patients with TNBC were treated with weekly paclitaxel plus liposomal doxorubicin (once a week for 18 weeks) every 3 weeks and were randomized to receive weekly carboplatin (area under the time-concentration curve [AUC]=2) or not. The pCR (ypT0ypN0) rate was 16\% higher with the addition of carboplatin (53.2\% vs $36.9 \%$ ) ( $\mathrm{P}=0.005)$. The toxicity was also significantly higher, with 53\% discontinuation (41\% with AUC reduced to 1.5). Data on the BRCA mutation are not yet available in order to assess the correct role of carboplatin.

\section{The adjuvant setting}

Studies in adjuvant setting also confirmed the activity and relevance of taxanes in TNBCs. Hayes et al retrospectively analyzed the histological samples of 52 patients enrolled in the study in order to evaluate the role of HER2 status on clinical end points. Patients were divided in four groups: endocrine receptor- and HER2-negative (TNBC); endocrine receptor-and HER2-positive; endocrine receptor-positive and HER2-negative; endocrine receptor-negative and HER2-positive. Adding taxanes/ cyclophosphane with anthracycline improved DFS both in HER2positive patients, independently from endocrine receptor status, and in TNBC patients. No clinical benefit was observed in HER2negative and endocrine receptor-positive tumors. This explorative analysis suggests that paclitaxel added to the adjuvant regimen significantly improves the outcome in TNBCs. The study of conducted on 52 patients, evaluated in the adjuvant setting the efficacy of AC followed by weekly or 3-weekly (q3w) docetaxel or paclitaxel. The results showed an improvement both in DFS and 5-year OS with weekly paclitaxel with respect to q3w paclitaxel. In TNBCs, the benefit of conventional weekly paclitaxel in term of DFS was $37 \%$ higher than the q3w regimen [23-26].

\section{The metastatic setting}

Conventional gemcitabine has a central role in the treatment of metastatic BC, based on several evidences of their benefits on clinical outcomes, such as OS, time to progression (TTP) $[25,26]$. Even if conventional gemcitabine demonstrated to be more active in endocrine receptor-negative tumors and are indicated in the first-line treatment of TNBC (although a specific benefit in this setting was not observed), it should be considered that they are commonly used in adjuvant therapy and cannot be challenged in case of short disease-free interval ( $<12$ months). Fan et al evaluated the efficacy of gemcitabine combined with cisplatin or capecitabine with docetaxel in the first-line treatment of patients with metastatic TNBC [27]. The ORR was significantly higher in patients treated with gemcitabine plus cisplatin than with docetaxel plus capecitabine (63\% vs $15.4 \%$ ) ( $\mathrm{P}=0.001$ ), as were the median PFS (10.9 vs 4.8 months) $(\mathrm{P}<0.001)$ and median OS $(32.8$ vs
21.5 months $)(\mathrm{P}=0.027)$, confirming the role of platinum in TNBC $[28,29]$.

The TNBC trial was based on the hypothesis that because BRCA1/2germline mutations produce BCs that have defects in homologous recombination DNA repair, carboplatin would be lethal to cells with germline and somatic mutations in BRCA1/2. In other words, carboplatin might be an especially good therapy in terms of exploiting the defect in homologous recombination DNA repair, and this is why patients with BRCA1/2 mutations were included with TNBC patients. The results showed no significant difference in response rates between carboplatin and gemcitabine in the overall patient group or in patients who received either agent as first-line therapy and then crossed over to the other agent as second-line treatment [28-30]. In conclusion, the main reason of failure in metastatic BC is resistance to the standard drugs, which can be intrinsic or acquired. Patients with disease progression or resistance could not have a cross-resistance with other drugs, such as capecitabine, gemcitabine, which demonstrated their efficacy in patients with advanced BC pretreated with carboplatin with gemcitabine.

\section{Conclusion}

TNBC is characterized by the absence of ER-, PgR-, and HER2-negativity: for this reason, the only therapeutic option is chemotherapy. Even if these tumors are chemo sensitive, as showed by the high pCR obtained with neoadjuvant therapy, metastatic patients have a short PFS; this the chemosensitivity does not translate in an improvement of PFS or OS, and the overall prognosis for these tumors is poor. The studies performed taxaneswithplatiniumbased chemotherapy demonstrated their efficacy in the treatment of TNBC in by setting neoadjuvant, and cyclophosphane with doxorubicin or docetaxel more effectively in adjuvant regimens, gemcitabine's with platinum was detected more metastatic triple negative breast cancer, international and national guidelines recommend the as possible active first-line therapeutic options for TNBC.

\section{References}

1. Navruzov SN, Khodzhaev AV, Khudaykulov AT (2013) Mammary cancer Problems of early diagnosis and prevention Guidelines.

2. Petrova GV, Gretsova OP, Starinsky VV (2011) Characteristics and methods for calculating statistical indicators used in oncology, Russia, p. 39.

3. Khudaykulov AT, Khudaykulov TK (2015) Socio-economic consequences of mortality of breast cancer in Uzbekistan. Malignant tumors, Uzbekistan, pp. 53-56.

4. Khudaykulov TK, Khudaykulov AT (2013) Epidemiological aspects of breast cancer in Uzbekistan. Bulletin of the Tashkent Medical Academy, Uzbekistan, pp. 95-97.

5. Ferlay J, Bray F, Pisani P, Parkin DM (2010) GLOBOCAN 2008: Cancer Incidence, Mortality and Prevalence Worldwide. IARC Cancer Base No.10.

6. Ferlay J, Soerjomataram I, Ervik M (2013) Cancer incidence and mortality worldwide: IARC cancer base no. 11: [Internet]. International Agency for Research on Cancer. 
7. Cardoso F, Harbeck N, Fallowfield L, Kyriakides S, Senkus E (2016) ESMO guidelines working group locally recurrent or metastatic breast cancer in uzbekistan: ESMO clinical practice guidelines for diagnosis, treatment and follow-up. Ann Oncol 23(Suppl 7): 11-19.

8. Nicolis F (2014) Numeridel Cancro in Italia. Associazione Italiana di Oncologia Medica, Milan, Italy.

9. DeRujters TC, Veeck J, DeHoon JPJ, Van Engeland M, Tjan Heijnen VC (2011) Characteristics of triple-negative breast cancer. J Cancer Res ClinOncol 137(2): 183-192.

10. Chacón RD, Costanzo MV (2010) Triple-negative breast cancer. Breast Cancer Res 12(Suppl 2): 3

11. Hudis CA, Gianni L (2011) Triple-negative breast cancer: An unmet medical need. Oncologist 16(Suppl 1): 1-11.

12. Rapoport BL, Nayler S, Demetriou GS, Moodley SD, Benn CA (2014) Triple-negative breast cancer pathologic diagnosis and current chemotherapy treatment options. Oncol Hematol Rev 10(1): 25-32.

13. Sørlie T, Perou CM, Tibshirani R, Aas T, Geisler S, et al. (2001) Gene expression patterns of breast carcinomas distinguish tumor subclasses with clinical implications. Proc Natl Acad Sci USA 98(19): 10869-10874.

14. Veer LJV, Dai H, Vande Vijver MJ (2002) Gene expression profiling predicts clinical outcome of breast cancer. Nature 415(6871): 530-536.

15. Hortobagyi GN, Hayes D, Pusztai L (2002) In: Johnson DH (Ed.), Integrating newer science into breast cancer prognosis and treatment: Molecular predictors and profiles. American Society of Clinical Oncology, Florida, USA.

16. Ayers M, Symmans WF, Stec J, Damokosh AI, Clark E, et al. (2004) Gene expression profiles predict complete pathologic response to neoadjuvant paclitaxel and fluorouracil, doxorubicin, and cyclophosphamide chemotherapy in breast cancer. J Clin Oncol 22(12): 2284-2293.

17. Dent R, Trudeau M, Pritchard KI, Hanna WM, Kahn HK, et al. (2007) Triple-negative breast cancer: Clinical features and patterns of recurrence. Clin Cancer Res 13(15 Pt 1): 4429-4434.

18. Lehmann BD, Bauer JA, Chen X, Sanders ME, Chakravarthy AB, et al. (2011) Identification of human triple-negative breast cancer subtypes and preclinical models for selection of targeted therapies. J Clin Invest 121(7): 2750-2767.

19. Lehmann BD, Pietenpol JA, Tan AR (2015) Triple-negative breast cancer:
Molecular subtypes and new targets for therapy. Am Soc Clin Oncol Educ Book 35: 31-39.

20. Rouzier R, Perou CM, Symmans WF, Ibrahim N, Cristofanilli M, et al. (2005) Breast cancer molecular subtypes respond differently to preoperative chemotherapy. Clin Cancer Res 11(16): 5678-5685.

21. Tobin NP, Harrell JC, Lövrot J, Egyhazi Brage S, Frostvik Stolt M, et al. (2014) Molecular subtype of breast cancer metastases significantly influences patient post-relapse survival. Ann Oncol 26(1): 81-85.

22. Dent RA, Mainwaring PN, Tan TJY, Barbier S, Javier Cortes, et al. (2015) Survival in triple-negative breast cancer (TNBC): Evidence from the SEER database 2010-2011. J ClinOncol.

23. NCCN (2014) National Comprehensive Cancer Network (NCCN) Clinical practice guidelines in oncology breast cancer version 2. PA: national comprehensive cancer network, Washington, USA.

24. Associazione Italiana di OncologiaMedica (AIOM) (2013) Linee Guida: neoplasiedella mammella. Associazione italiana di oncologia medica, Milan, Italy.

25. Cortazar P, Zhang L, Untch M, Keyur M, Joseph P Costantino, et al. (2014) Pathological complete response and long-term clinical benefit in breast cancer: the CT Neo BC pooled analysis. Lancet 384(9938): 164-172.

26. Cortazar P, Geyer CE, Gianni L, Cameron D, Minckwitz GV (2015) Pathological complete response in breast cancer- Authors' reply. Lancet 385: 114-115.

27. Minckwitz G, Untch M, Nüesch E, Loibl S, Kaufmann M, et al. (2011) Impact of treatment characteristics on response of different breast cancer phenotypes: pooled analysis of the German neo-adjuvant chemotherapy trials. Breast Cancer Res Treat 125(1): 145-156.

28. Minckwitz G, Untch M, Blohmer JU, Costa SD, Eidtmann H, et al. (2012) Definition and impact of pathologic complete response on prognosis after neoadjuvant chemotherapy in various intrinsic breast cancer subtypes. J ClinOncol 30(15): 1796-1804.

29. Liedtke C, Mazouni C, Hess KR, André F, Tordai A, et al. (2008) Response to neoadjuvant therapy and long-term survival in patients with triplenegative breast cancer. J Clin Oncol 26(8): 1275-1281.

30. Guarneri V, Broglio K, Kau SW, Massimo Cristofanilli, Aman UB, et al (2006) Prognostic value of pathologic complete response after primary chemotherapy in relation to hormone receptor status and other factors. J Clin Oncol 24(7): 1037-1044.

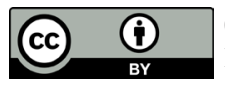

Creative Commons Attribution 4.0 International License

For possible submissions Click Here
Submit Article

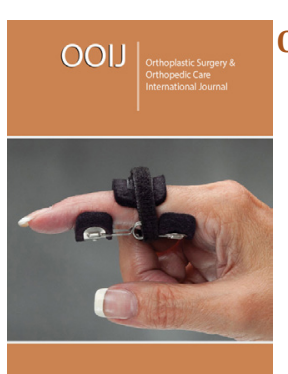

Orthoplastic Surgery \& Orthopedic Care International Journal

\section{Benefits of Publishing with us}

- High-level peer review and editorial services

- Freely accessible online immediately upon publication

- Authors retain the copyright to their work

- Licensing it under a Creative Commons license

- Visibility through different online platforms 